\title{
Brief Strategic Therapy vs Cognitive Behavioral Therapy for the Inpatient and Telephone-Based Outpatient Treatment of Binge Eating Disorder: The STRATOB Randomized Controlled Clinical Trial
}

\author{
Gianluca Castelnuovo $^{1,2, *}$, Gian Mauro Manzoni ${ }^{1,3}$, Valentina Villa ${ }^{1}$, Gian Luca Cesa ${ }^{1}$ and \\ Enrico Molinari ${ }^{1,2}$ \\ ${ }^{1}$ Istituto Auxologico Italiano IRCCS, Psychology Research Laboratory, Ospedale San Giuseppe, Verbania, Italy \\ ${ }^{2}$ Department of Psychology, Catholic University of Milan, Italy \\ ${ }^{3}$ Department of Psychology, University of Bergamo, Italy
}

\begin{abstract}
This paper describes the results of the STRATOB (Systemic and STRATegic psychotherapy for OBesity) study, a two-arm randomized controlled clinical trial (RCT) comparing Brief Strategic Therapy (BST, Nardone or Arezzo model) with the gold standard CBT (Cognitive Behavioral Therapy) for the inpatient and telephone-based outpatient treatment of obese people with Binge Eating Disorder (BED) seeking treatment for weight reduction. Primary outcome measure of the randomized trial was change in the Global Index of the Outcome Questionnaire (OQ 45.2). Secondary outcome measures were BED remission (weekly binge episodes < 2) and weight loss. Data were collected at baseline, at discharge from the hospital (c.a. 1 month after) and after 6 months from discharge.. No significant difference between groups (BST vs CBT) was found in the primary outcome at discharge. However, a greater improvement was seen in the BST vs the CBT group $(\mathrm{P}<.01)$ in the primary outcome at 6 months. About secondary outcomes, no significant difference between groups were found in weight change both at discharge and at 6 months. Notably, a significant association emerged between treatment groups and BED remission at 6 months in favor of BST (only 20\% of patients in BST group reported a number of weekly binge episodes $>2$ vs $63.3 \%$ in CBT group).
\end{abstract}

Keywords: Brief strategic therapy - BST, cognitive behavioural therapy - CBT, binge-eating disorder - BED, clinical psychology, obesità, telecare, psychotherapy.

\section{INTRODUCTION}

Obesity constitutes one of the most important medical and public health problems of our time [1]. It is considered as a chronic pathology and is widely recognized as a risk factor for many medical complications such as cardiovascular, orthopedic, pneumological and endocrinological diseases [2-8]. Overweight and obesity is also linked with Binge Eating Disorder (BED). "Binge eating disorder is characterized by frequent and persistent episodes of binge eating accompanied by feelings of loss of control and marked distress in the absence of regular compensatory behaviors" (p. 94. [9]). Functional interventions for significantly reduce weight, maintain weight loss and manage associated pathologies like BED are tipically combined treatment options (dietetic, nutritional, physical, behavioral, cognitive-behavioral, pharmacological, surgical). Significant difficulties with regard to availability, costs, treatment adherence and long-term efficacy are present [10]. Moreover, most overweight and obese individuals regain about one third of the weight lost with treatment within 1 year [11] and they are typically back to baseline in 3 to 5 years [12-14].

*Address correspondence to this author at the Psychology Research Laboratory, San Giuseppe Hospital, Istituto Auxologico Italiano, Via Cadorna, 90, 28824 Piancavallo (VB), Italy; Tel: 0039323 514338;

Fax: 0039323 514338; E-mail: gianluca.castelnuovo@auxologico.it
Treatment for BED is directed towards either the physical or psychopathological impairments and Cognitive behavior therapy (CBT) and Interpersonal Psychotherapy (IPT) are psychotherapies for BED indicated to target the eating disorder [9]. Particularly CBT is the therapeutic approach indicated both in inpatient and in outpatient settings for BED [15-27]. In recent years systemic and systemic-strategic psychotherapies have been implemented to treat patients with obesity and BED involved in familiar problems [26-30]. Particularly, a brief protocol for the systemic-strategic treatment of BED, using overall the strategic dialogue, has been developed by Nardone and Portelli in the Handbook of Brief Strategic Therapy (BST) [31].

Furthermore, telemedicine, a new promising low cost method, has been used for obesity with BED in outpatient settings in order to avoid relapse after the inpatient step of treatment and to keep on a continuity of care with the involvement of the same clinical inpatient team [32-35].

For these reasons, we developed STRATOB (Systemic and STRATegic psychotherapy for OBesity), a comprehensive two-phase stepped down program enhanced by telepsychology for the mid-term treatment of obese people with BED seeking intervention for weight loss. The core aspects of STRATOB are the inpatient intensive treatment and the continuity of care at home using a low-level of telecare (mobile phones). Many treatments delivered using technologies, 
such as web-sites, e-mails, chat lines, videoconferences, UMTS-based mobile-phones and telephones, could be a valid integration to traditional psychotherapy reducing expensive and time-consuming clinical visits and improving adherence to prescribed psychological, dietetic and medical treatments through extensive monitoring and support $[10,12$, 36-41].

This paper describes the design and the results of the STRATOB study, a two-arm randomized controlled clinical trial (RCT). The aims of this study are to compare the effectiveness of the BST [31, 42, 43] with the gold standard CBT $[26,44-50]$ in a inpatient and telephone-based outpatient program for a sample of obese people with BED seeking treatment for weight reduction.

\section{MATERIALS AND METHODOLOGY}

\section{Study Design and Protocol}

CBT and BST have been compared in a randomized controlled clinical trial. Participants have been randomly allocated in 2 groups:

1) CBT group: inpatient treatment (diet, physical activity, dietitian counseling, 8 sessions of CBT) plus 8 outpatient telephone-based sessions of CBT-oriented psychological support and monitoring with the same CBT inpatient psychotherapists;

2) BST group: inpatient treatment (diet, physical activity, dietitian counseling, 8 sessions of BST) plus 8 outpatient telephone-based sessions of BST-oriented psychological support and monitoring with the same BST inpatient psychotherapists;

The Medical Ethics Committee of Istituto Auxologico Italiano approved the study protocol and the Informed Consents.

The treatment program considered in this study has a total duration of 7 months and consists of two stepped down phases: in-patient (1 month) and out-patient (the following 6 months). During the in-patient phase, participants undergo an intensive four-week hospital-based and medicallymanaged program for weight reduction and rehabilitation. Along this period, participants live in a medical hospital-like environment located on a mountain highland and far away from towns and cities. Visits from parents are allowed only in the afternoon. All patients are placed on a hypocaloric nutritionally balanced diet tailored to the individual after consultation with a dietitian (energy intake around $80 \%$ of the basal energy expenditure estimated according to the Harris-Benedict equation and a macronutrient composition of about $16 \%$ proteins, $25 \%$ fat and $59 \%$ carbohydrates). Furthermore, they receive nutritional counseling provided by a dietitian, psychotherapy provided by psychologists-psychotherapists (well trained in CBT and BST approaches) and have physical activity training provided by a physiotherapist.

Nutritional rehabilitation program aims to improve and promote change in eating habits and consists of both individual sessions (dietary assessment, evaluation of nutrient intake and adequacy, nutritional status, anthropometric, eating patterns, history of overweight, readiness to adopt change) and group sessions (45 minutes each twice a week) including: information on obesity and related health risks, setting of realistic goals for weight loss, healthy eating in general, general nutrition and core food groups, weight management and behavior change strategies for preventing relapse).

Psychotherapy is provided twice a week in a face-to-face setting. CBT individual sessions, lasting 45 minutes each, are mainly based on the cognitive-behavioral approach described by Cooper and Fairburn [50] and emphasize the techniques of self-monitoring, goal setting, time management, prompting and cueing, problem solving, cognitive restructuring, stress management and relapse prevention. BST individual sessions, lasting 45 minutes each, are mainly based on the brief strategic approach described by Nardone and Portelli [31] and emphasize the techniques of working on "attempted solutions" (such as keeping control by abstaining from food and continuously striving to exert self-control with a subsequent loss of control), using reframing maneuvers, inducing fear of fasting rather then bingeing, understanding what is maintaining and worsening the problem, etc. (p. 105-105, [31]).

Physical activity takes place once a day except for weekend and consists of group programs (20 subjects) based on postural gymnastics, aerobic activity and walks in the open. Inpatients with specific orthopedic complications carry out individual activities planned by physiotherapists and articulated in programs of physical therapy, assisted passive and active mobilization and isokinetic exercise.

Low to moderate weight losses are expected at the end of the in-patient phase, but it is important to note that weight loss is not the primary goal of the in-patient program and each patient is made clear about this point at the very beginning of the treatment. Beyond the medical management of metabolic risk factors for health such as type 2 diabetes, developing a sense of autonomy and competence are the primary purposes of the in-hospital interventions. Patients are afforded the skills and tools for change and are supported in assigning positive values to healthy behaviors and also in aligning them with personal values and lifestyle patterns.

Just after discharge, participants have 4 telephone calls with their psychotherapists along 2 months. From the 3 rd to the 6th month sessions are scheduled every 30 days. During tele-sessions, psychotherapists test the outpatients' progress, their mood, the maintenance of the "good alimentary and physical activity habits", the loss/increase of weight and ask about critical moments. Some research indicates that changes in behavior (eating and exercise) often follow discrete moments which have been variably described as life events, life crises, teachable moments or epiphanies [51]. Life events can lead to weight loss but also to weight gain and qualitative research shows that it is not the event per se that results in behavior change but the ways in which this event is appraised and interpreted by the individual [52]. The psychotherapists (both CBT and BST) have thus the opportunity to discuss with the outpatients about the significant events during the telephone calls and cognitively reconstruct dysfunctional appraisals in functional ways.

In particular, phone-sessions with the psychotherapists aim to consolidate strategies and abilities acquired during the in-patient phase, to improve self-esteem and self-efficacy, to support motivation, to prevent relapse and to provide problem-solving and crisis counseling. 
As described, in the outpatient phase of the STRATOB program great relevance is given to the psychotherapistpatient relationship as an important medium and vehicle of change [53]. After discharge, out-patients begin to experience the autonomy and competence to change they develop during the in-patient phase and inevitably face resistances and barriers. Thanks to telephone calls, out-patients are supported by the psychotherapists who attended them during the in-hospital phase in exploring resistances and barriers they experience and in finding functional pathways to cope. Furthermore, out-patients are helped to experience mastery in terms of the health behavior change that needs to be engaged.

\section{Study Population}

\section{Recruitment of the Study Population}

One hundred consecutive female inpatients with obesity and BED in the age range of 18-65 years who were referred to the single clinical center (Saint Joseph Hospital - Istituto Auxologico Italiano) for weight-loss treatment have been asked and screened for admission to the study. Patients were sequentially recruited in 2008. Of these, 37 did not fulfill inclusion criteria and 7 declined to partecipate for other reasons (e.g., time constraints, involvement in other treatments). Finally, 60 patients (mean age: $46.05 \pm 10.54$ years; mean weight: $106.95 \pm 6.94 \mathrm{~kg}$ ) entered the treatment phase.

\section{Inclusion and Exclusion Criteria}

Inpatients were eligible when they met the following inclusion criteria at the admission to the hospital: 1) age between 18 and 65 years; 2) obesity according to the WHO criteria (BMI $\geq 30)$; 3) BED (DSM-IV-TR criteria) and 4) written and informed consent to participate. Exclusion criteria for the study are: 1) other severe psychiatric disturbance diagnosed by DSM-IV-TR criteria; 2) concurrent medical condition not related to obesity. SCID (Structured Clinical Interview for DSM-IV-TR Disorders) I and II [54] were used as screening tools for psychiatric disorders and were administered by an independent clinical psychologist as part of his work.

\section{Randomization Procedure}

All participants were randomly assigned to the CBT or BST group. The randomization scheme have been generated by using the Web site Randomization.com (www. randomization.com). Randomization took place after the baseline measurements.

\section{Psychotherapists and Treatment Fidelity}

Treatments were delivered by 4 experienced and chartered psychotherapists from diverse backgrounds with specific training in CBT and BST. Psychotherapists received monthly supervision by senior chartered psychotherapists to ensure competent and uniform treatment delivery.

\section{Patient Assessment and Measurements}

Primary outcome measure of the randomized trial is change in the Global Index of the Outcome Questionnaire (OQ 45.2). Secondary outcome measures are BED remission defined as a number of weekly binge episodes $<2$ and weight loss. Data were collected at baseline, at discharge from the hospital (c.a. 1 month after) and after 6 months from discharge.

Weight was assessed with the participant in lightweight clothing with shoes removed on a balance beam scale. Data collected at 6-month follow-up were collected through telesessions.

\section{Psychological and Behavioral Assessment}

Participants completed the Outcome Questionnaire (OQ 45.2) - Italian translation and validation [55, 56] at entry to the study and at discharge from the hospital with a selfreport procedure. At 6-month follow-up data have been collected via telephone during the tele-sessions.

The OQ 45.2 is an self report questionnaire developed by Michael Lambert in 1996 [57]. The OQ 45 items version is a measure of outcome and it is designed in order to collect repeated measures of patient progress during psychotherapy and after its conclusion. This instrument is one of the most used in psychotherapy research in the U.S. [58]. The OQ 45.2 is composed by 45 items that form 3 scales: Symptom Distress (SD), Interpersonal Relations (IR) e Social Role (SR), and a Global Index. The OQ 45.2 is proposed as a brief screening and outcome scale that tries to assess the subjective experience of a person, as well as the way he or she functions in the world [57]. The choice of this questionnaire has to take into account the need to measure a change in a short time (1 month of in-patient treatment) due to psychotherapeutic activities. The Italian version of the OQ-45.2 appears promising as a measure of general psychological distress, and it could be used to measure the psychotherapy outcome in routine clinical practice. So the Outcome Questionnaire- 45 was developed as a brief measure of client progress in psychotherapy to address the need for accountability in the era of managed care and to improve upon extant outcome measures.

Number of weekly binge episodes was assessed with a self-report procedure at entry to the study and at discharge from the hospital. At 6-month follow-up, data have been collected via telephone during the tele-sessions.

\section{Sample Size Calculation and Statistical Analysis}

Due to the novelty of the application of BST in BED treatment, no other RCTs including BST have been carried out. So this RCT is similar to a pilot study, typically defined as small scale preliminary study, and according to Lackey and Wingate [59], a pilot work may use at least the $10 \%$ of the sample required. Using an A-priori Sample Size Calculator for Student's t-Test (G*Power 3.1.2 software), 64 participants per group were needed to detect a difference with an estimated Effect Size (Cohen's d) of 0.5, an alpha of 0.05 two-sided, a desired statistical power of 0.80 . We decided not to make assumptions on sidedness not having previous results about comparison between CBT and BST. Taking into account the need of at least $10 \%$ of the sample required (128 individuals, $n=64$ per group), 60 persons $(n=30$ per group) were considered enough.

Descriptive statistics (means $\pm \mathrm{SD}$ ) have been used to describe the study sample with regard to baseline characteristics (Table 1). The independent samples $t$ test was used to examine between-group differences in $\mathrm{OQ}$ variables and 
Table 1. Baseline Participant Characteristics by Treatment Group

\begin{tabular}{|l|c|c|c|}
\hline \multicolumn{1}{|c|}{ Characteristic } & CBT Group $(\mathbf{n}=\mathbf{3 0})$ & BST Group $(\mathbf{n}=\mathbf{3 0})$ & Total $(\mathbf{N}=\mathbf{6 0})$ \\
\hline \hline Age, $\mathbf{y} *$ & $46,2(10,5)$ & $45,9(10,76)$ & $46,05(10,54)$ \\
\hline Female sex & 30 & 30 & 60 \\
\hline Weight, Kg * & $107,37(6,83)$ & $106,53(7,14)$ & $106,95(6,95)$ \\
\hline OQ 45.2 Symptom Distress (SD) * & $48,47(8,42)$ & $52,13(11,19)$ & $25,3(10)$ \\
\hline OQ 45.2 Interpersonal Relations (IR)* & $24,40(5,8)$ & $25,93(7,61)$ & $18,95(4,55)$ \\
\hline OQ 45.2 Social Role (SR)* & $19,50(4,47)$ & $18,4(4,64)$ & $94,42(10,73)$ \\
\hline OQ 45.2 Global Index * & $92,37(11,01)$ & $96,47(10,22)$ & $2,82(0,77)$ \\
\hline Number of weekly binge episodes * & $2,83(0,74)$ & $2,8(0,8)$ & \\
\hline
\end{tabular}

*Data are given as mean (SD).

weight at all time-points. Change scores were also examined. Chi-square was used to test the association between treatment groups and number of weekly binge episodes $\geq 2$ at 6 months. Corrected effect sizes (Hedges) and 95\% Confidence Interval were calculated for both between-group and with-in group differences. All data analyses were performed using the Statistical Package for the Social Sciences (version 16.0; SPSS, Inc., Chicago, IL).

\section{RESULTS}

There were no differences between CBT and BST on any demographic, diagnostic and other clinical variable at pretreatment (Table 1).

No significant between-group difference was found in change scores from baseline to discharge in any variables (Table 2). At 6 months, OQ 45.2 Global Index as well as the OQ 45.2 Symptom Distress (SD) change scores from discharge were higher in the BST vs the CBT group $(\mathrm{p}<0,000)$ (Table 2 and Fig. 1).

Table 3 shows the within-group changes for all the variables at all time-points. BST group had statistically significant improvements from baseline to discharge as well as from discharge to 6-month follow-up in all continuous outcomes. CBT group had significant improvements on all the variables but not in the OQ 45.2 Interpersonal Relations (IR) change from Baseline to Discharge, in the OQ 45.2 Interpersonal Relations (IR) change from Discharge to 6 months and in Percentage of weight loss from discharge to 6 months ( $95 \%$ confidence intervals of mean changes include zero).

With respect to absolute weight loss, no significant between-group difference was found in weight loss from baseline to discharge and from discharge to 6 months (Fig. 2). A significant association was found between treatment groups and BED remission at 6 months (Fig. 3). In the BST group, only $20 \%$ of participants vs $63,3 \%$ in the CBT group $(\mathrm{p}=0,001)$ reported a number of weekly binge episodes over the cut-off $(>2)$ for the BED diagnosis.

\section{DISCUSSION AND LIMITATIONS}

Due to the novelty of Brief Strategic Therapy (BST) in the treatment of Binge Eating Disorder, no well defined hypothesis have been established about the possible contribu- tion of this kind of therapy in comparison with Cognitive Behavior Therapy (CBT), the "gold standard" psychological treatment for BED. With the development of a brief systemic-strategic clinical protocol for treatment of BED [31], new interventions in the field of eating disorders are available and have to be evaluated. Systemic and systemicstrategic psychotherapies such as BST traditionally focus on systemic-family problems and contexts and these areas of interventions could represent a real added value in comparison with CBT.

Moreover, BST sessions emphasize the techniques of working on "attempted solutions", using reframing maneuvers, inducing fear of fasting rather then bingeing, understanding what is maintaining and worsening the problem, etc. (p. 105-106, [31]). This focus on "attempted solutions", that in the typical BED are related to dysfunctional habits such as "trying to keep control by abstaining from food and continuously striving to exert self-control with a subsequent loss of control", could explain the observed superiority of BST vs CBT in producing a significant remission of weekly binge episodes at 6 months. During the inpatient phase patients did not cope with the binge temptations due to the lack of them in a safe place like the hospital. However, after discharge, patients went back home and faced again with their temptations. BST suggestions about managing binge eating was proved to be more effective in preventing relapse than CBT after 6 months from discharge.

The other significant difference in favor of BST, i.e. the higher improvement in the OQ 45.2 Global Index at 6 months, is probably due to the focus of BST on symptoms reduction (in particular anxiety and depression) in short time (traditionally 6-months). Indeed, a significant difference between CBT and BST was found also in the OQ 45.2 Symptom Distress (SD) scale, where greater improvements were seen in the BST vs the CBT group $(\mathrm{p}<.01)$.

The real therapeutic impact of CBT and BST could have been significantly reduced by the use of an unusual setting in the second phase of the trial. Even if telecare could represent a great opportunity for the continuity of treatments "moving the healthcare where it really needs", the limitations of nonverbal communications, due to the use of telephone sessions, could have influenced the CBT and BST protocols 
Table 2. Differences Between CBT and BST Groups Considering Primary Outcome Measure (Change in the Global Index and Other Sub-Indexes of the OQ 45.2) and Secondary Outcome Measures (Percentage of BED Patients Remitted Considering the Number of Weekly Binge Episodes and the Weight Loss) at Discharge from the Hospital and After 6 Months

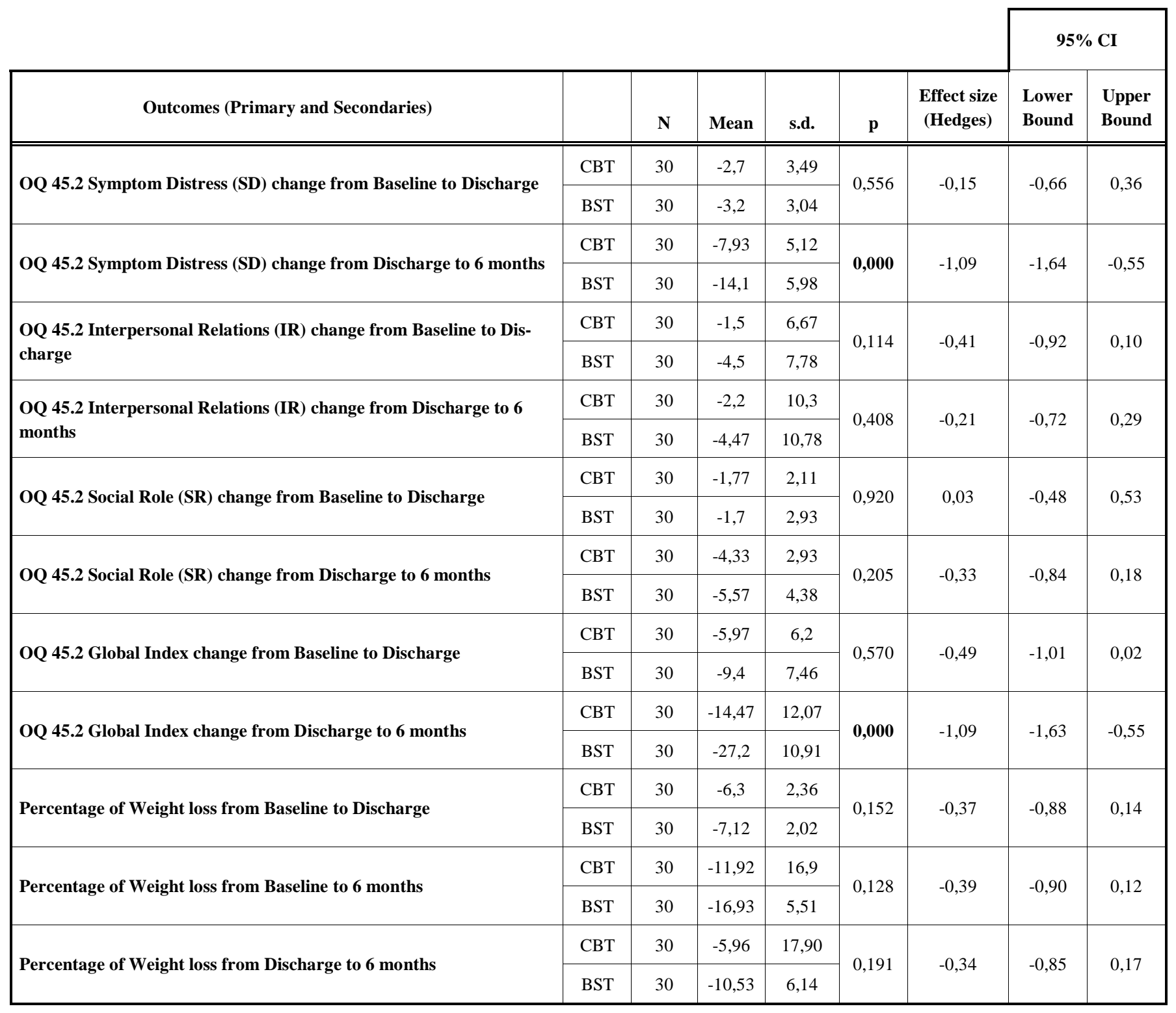

Table 3. Within-Group Changes of Primary Outcome Measure (OQ 45.2 Global Index) and Secondary Outcome Measures (OQ 45.2 Sub-Indexes and Weight Loss) at Discharge from the Hospital and after 6 Months by Treatment Group (CBT and BST)

\begin{tabular}{|l|c|c|c|c|c|c|}
\hline \multicolumn{2}{|l|}{} & \multicolumn{2}{|c|}{$\begin{array}{c}\text { Confidence Interval of } \\
\text { the Difference }\end{array}$} \\
\hline \multicolumn{1}{|c|}{ Outcomes (Primary and Secondaries) } & Group & N & Mean & Std. Deviation & Lower Bound & Upper Bound \\
\hline \hline \multirow{2}{*}{$\begin{array}{l}\text { OQ 45.2 Symptom Distress (SD) change from Baseline to } \\
\text { Discharge }\end{array}$} & CBT & 30 & $-2,7$ & 3,49 & $-3,95$ & $-1,45$ \\
\cline { 2 - 7 } & BST & 30 & $-3,2$ & 3,04 & $-4,29$ & $-2,11$ \\
\hline $\begin{array}{l}\text { OQ 45.2 Symptom Distress (SD) change from Discharge } \\
\text { to 6 months }\end{array}$ & CBT & 30 & $-7,93$ & 5,12 & $-9,77$ & $-6,10$ \\
\cline { 2 - 7 } $\begin{array}{l}\text { OQ 45.2 Interpersonal Relations (IR) change from Base- } \\
\text { line to Discharge }\end{array}$ & BST & 30 & $-14,1$ & 5,98 & $-16,24$ & $-11,96$ \\
\cline { 2 - 7 } & BST & 30 & $-1,5$ & 6,67 & $-3,89$ & $\mathbf{0 , 8 9}$ \\
\hline
\end{tabular}


Table 3. contd....

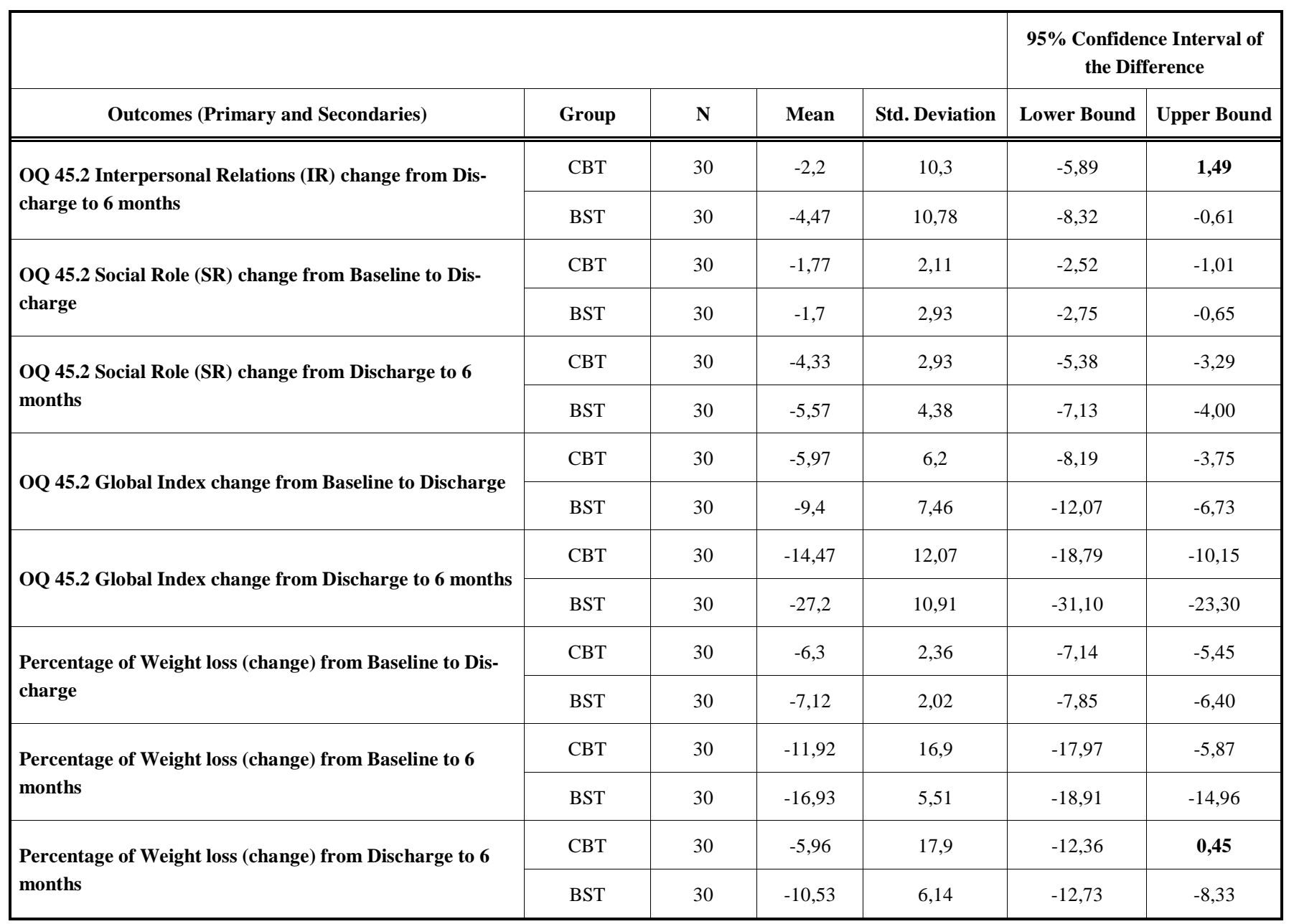

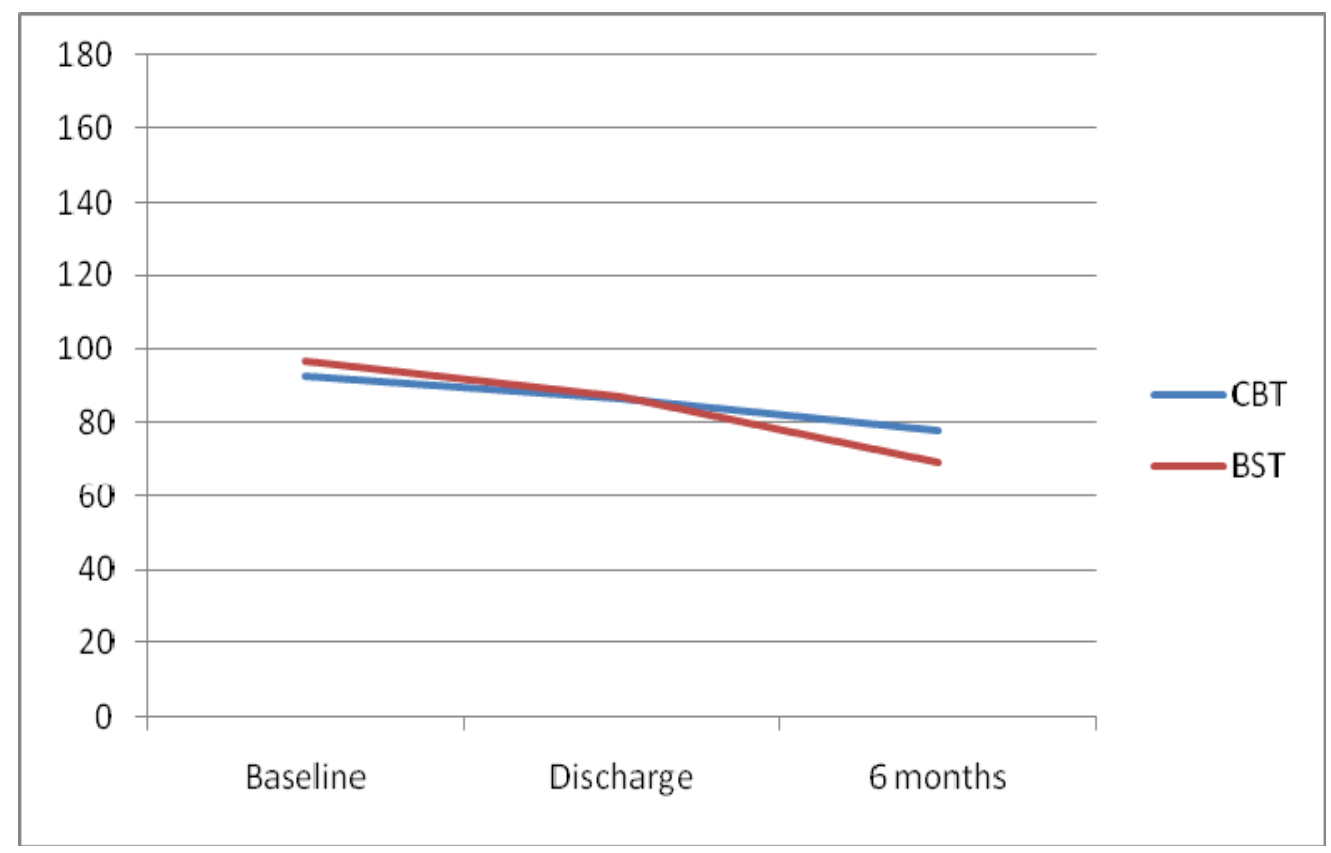

Note: A significant difference $(p<.01)$ between CBT and BST were found only at 6 months

Fig. (1). Global Index of the OQ 45.2 at baseline, at discharge from the hospital and after 6 months by Treatment Group (CBT and BST)*. 


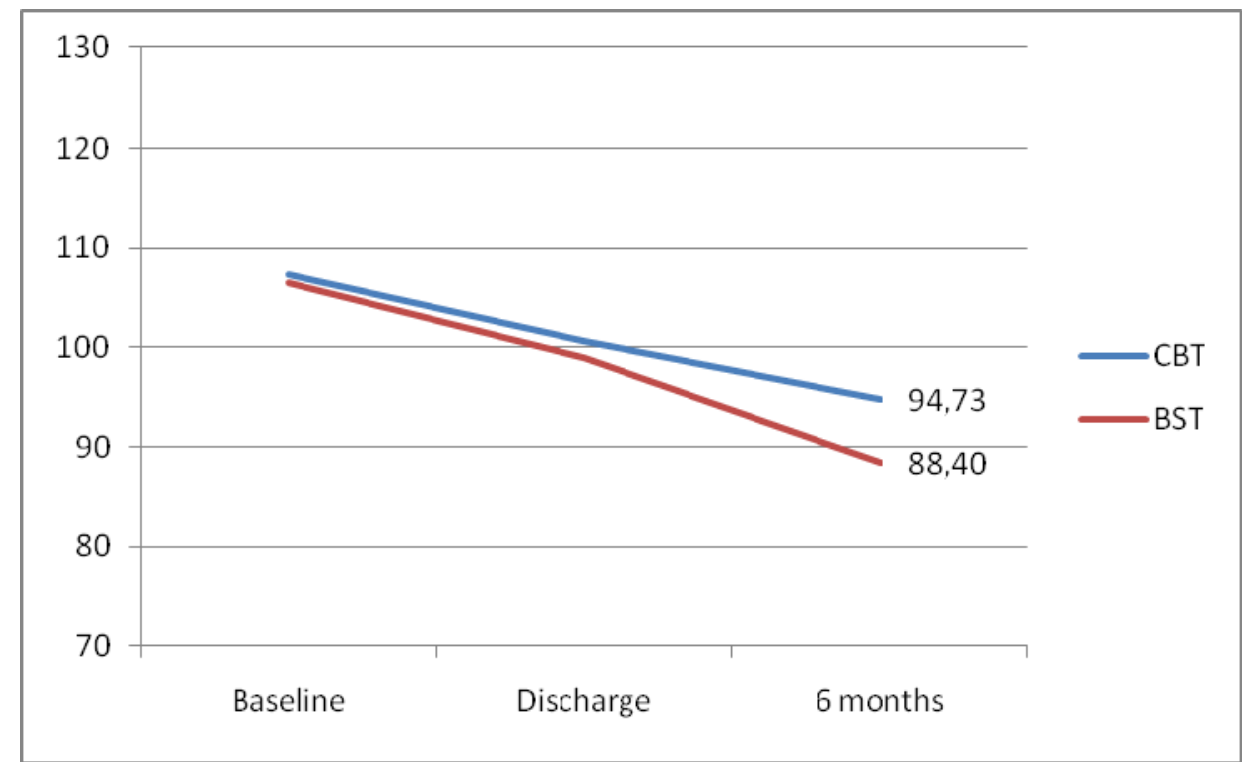

Note: No significant differences between CBT and BST were found at the 3 time-points

Fig. (2). Change in weight (weight loss) at baseline, at discharge from the hospital and after 6 months by Treatment Group (CBT and BST)*.

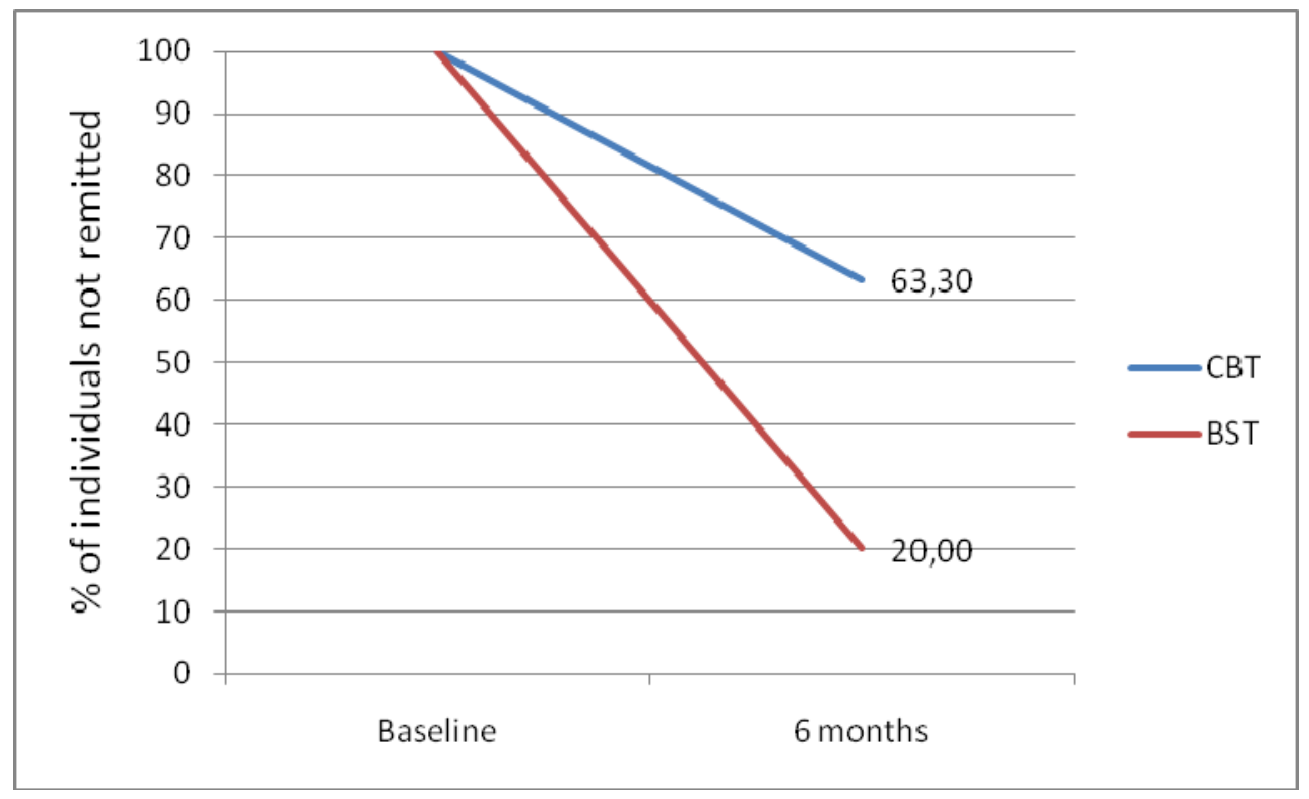

Note: BED remission was defined as a number of weekly binge episodes $<2$.

Fig. (3). Percentage of individuals not remitted at 6 months by Treatment Group (CBT and BST).

in different ways that have to be investigated in future researches.

Another study limitation concerns the low number of psychometric tools used in the evaluation of change. Even if the the OQ 45.2 is designed to collect repeated measures of patient progress during psychotherapy and after its conclusion, no specific scale or questionnaire was used to assess the eating dysfunctional area of patients. Number of weekly binge episodes was used as a simple index for BED remission and data showed that BST was more effective than CBT in preventing relapse, but in future trials the more reliable number of weekly binge days (and not the number of weekly binge episodes, as suggested by Wilson and Sysko [60]) should be used.

A final limitation is small sample size. Even if this is the first study that compared CBT with BST in the treatment of $\mathrm{BED}$, larger clinical trials are needed in order to evaluate both the effectiveness of integrated psychotherapies (such as the traditional CBT combined with BST or IPT) and the use of new technologies in the clinical field (from the "low technology" telephone to the "medium technology" clinically oriented SMS, MMS, e-mail, chat, web sources, till the "high technology" web 2.0 or virtual and augmented realities) [6167]. 
Given that the STRATOB trial was a single-centre randomized controlled study, generalization of research findings is limited. Future multi-centre trials should extend both the use of alternative models of psychological support or psychotherapy, i.e. BST, and the implementation of new technologies in order to find the best combination of the variables involved (psychotherapeutic model, level of technology, number of sessions, etc.).

Considering that traditional interventions for BED patients with obesity typically fails because of low compliance with clinical programs, continuous monitoring and psychological support are needed. This study improved the evidence-based knowledge of how CBT and BST may enhance the long-term efficacy of clinical interventions for BED and weight loss also using telecare settings. Due to the growing development of technologies in the clinical applications, we are moving towards a new "health care everywhere" approach, where clinicians could provide their treatments in both traditional clinical settings (public and private hospitals, clinics, services, etc.) and innovative clinical settings (remote outpatients' clinics, tele-health and e-health based settings).

\section{ACKNOWLEDGEMENTS}

The STRATOB Project was a preliminary low technology-based part of the more comprehensive high-technology based TECNOB Project supported by the "Compagnia di San Paolo" private foundation. No technological partners are involved in this STRATOB Project.

\section{REFERENCES}

[1] WHO. Definition and Diagnosis of Diabetes Mellitus and Intermediate Hyperglycemia. Report of a WHO/IDF consultation 2006.

[2] Pi-Sunyer X. The medical risks of obesity. Postgrad Med 2009; 121: 21 .

[3] Gnacinska M, Malgorzewicz S, Stojek M, Lysiak-Szydlowska W, Sworczak K. Role of adipokines in complications related to obesity: a review. Adv Med Sci 2009; 54: 150.

[4] van Kruijsdijk RC, van der Wall E, Visseren FL. Obesity and cancer: the role of dysfunctional adipose tissue. Cancer Epidemiol Biomarkers Prev 2009; 18: 2569.

[5] McCallister JW, Adkins EJ, O'Brien JM, Jr. Obesity and acute lung injury. Clin Chest Med 2009; 30: 495.

[6] Shah N, Roux F. The relationship of obesity and obstructive sleep apnea. Clin Chest Med 2009; 30: 455.

[7] Beleigoli AM, Diniz MF, Ribeiro AL. Natriuretic peptides: linking heart and adipose tissue in obesity and related conditions--a systematic review. Obes Rev 2009; $10: 617$.

[8] Momeni A, Heier M, Bannasch H, Stark GB. Complications in abdominoplasty: a risk factor analysis. J Plast Reconstr Aesthet Surg 2009; 62: 1250 .

[9] Wilson GT, Wilfley DE, Agras WS, Bryson SW. Psychological treatments of binge eating disorder. Arch Gen Psychiatry 2010; 67: 94-101.

[10] Weinstein PK. A review of weight loss programs delivered via the internet. J Cardiovasc Nurs 2006; 21: 251.

[11] Hill JO, Thompson H, Wyatt H. Weight maintenance: what's missing? J Am Diet Assoc 2005; 105: S63.

[12] Wing RR, Tate DF, Gorin AA, Raynor HA, Fava JL. A selfregulation program for maintenance of weight loss. N Engl J Med 2006; 355: 1563.

[13] Jeffery RW, Drewnowski A, Epstein LH, et al. Long-term maintenance of weight loss: current status. Health Psychol 2000; 19: 5.

[14] Katan MB. Weight-loss diets for the prevention and treatment of obesity. N Engl J Med 2009; 360: 923.

[15] Schlup B, Munsch S, Meyer AH, Margraf J, Wilhelm FH. The efficacy of a short version of a cognitive-behavioral treatment followed by booster sessions for binge eating disorder. Behav Res Ther 2009; 47: 628 .
[16] Sarwer DB. Comment on: brief, four session group CBT reduces binge eating behaviors among bariatric surgery candidates (Ashton $\mathrm{K}$, et al. 2009;5:257-62). Surg Obes Relat Dis 2009; 5: 407.

[17] Ashton K, Drerup M, Windover A, Heinberg L. Brief, four-session group CBT reduces binge eating behaviors among bariatric surgery candidates. Surg Obes Relat Dis 2009; 5: 257.

[18] Tsiros MD, Sinn N, Brennan L, et al. Cognitive behavioral therapy improves diet and body composition in overweight and obese adolescents. Am J Clin Nutr 2008; 87: 1134.

[19] Munsch S, Roth B, Michael T, et al. Randomized controlled comparison of two cognitive behavioral therapies for obese children: mother versus mother-child cognitive behavioral therapy. Psychother Psychosom 2008; 77: 235.

[20] Shelley-Ummenhofer J, MacMillan PD. Cognitive-behavioural treatment for women who binge eat. Can J Diet Pract Res 2007; 68: 139.

[21] Eichler K, Zoller M, Steurer J, Bachmann LM. Cognitivebehavioural treatment for weight loss in primary care: a prospective study. Swiss Med Wkly 2007; 137: 489.

[22] Brownley KA, Berkman ND, Sedway JA, Lohr KN, Bulik CM. Binge eating disorder treatment: a systematic review of randomized controlled trials. Int J Eat Disord 2007; 40: 337.

[23] Vaidya V. Cognitive behavior therapy of binge eating disorder Adv Psychosom Med 2006; 27: 86.

[24] Grilo CM, Masheb RM, Wilson GT. Rapid response to treatment for binge eating disorder. J Consult Clin Psychol 2006; 74: 602.

[25] Fennig S. Can we treat morbid obese children in a behavioral inpatient program? Pediatr Endocrinol Rev 2006; 3 (Suppl 4): 590.

[26] Berkman ND, Bulik CM, Brownley KA, et al. Management of eating disorders. Evid Rep Technol Assess (Full Rep) 2006: 135: 1166.

[27] Wilson GT. Psychological treatment of eating disorders. Annu Rev Clin Psychol 2005; 1: 439

[28] Peterson Y. Family therapy treatment: working with obese children and their families with small steps and realistic goals. Acta Paediatr Suppl 2005; 94: 42.

[29] Molinari E, Baruffi M, Croci M, Marchi S, Petroni ML. Binge eating disorder in obesity: comparison of different therapeutic strategies. Eat Weight Disord 2005; 10: 154.

[30] Wilfley DE, Cohen LR. Psychological treatment of bulimia nervosa and binge eating disorder. Psychopharmacol Bull 1997; 33: 437.

[31] Nardone G, Portelli C. Knowing through changing: The Evolution of Brief Strategic Therapy: Crown House Publishing Limited 2005.

[32] Marks S, Shaikh U, Hilty DM, Cole S. Weight status of children and adolescents in a telepsychiatry clinic. Telemed $\mathrm{J}$ E Health 2009; 15: 970.

[33] Shaikh U, Cole SL, Marcin JP, Nesbitt TS. Clinical management and patient outcomes among children and adolescents receiving telemedicine consultations for obesity. Telemed J E Health 2008; 14: 434.

[34] Riva G, Alcaniz M, Anolli L, et al. The VEPSY UPDATED project: technical and clinical rationale. Stud Health Technol Inform 2002; 85: 395.

[35] Riva G, Alcaniz M, Anolli L, et al. The VEPSY updated project: virtual reality in clinical psychology. Cyberpsychol Behav 2001; 4 449.

[36] Tate DF, Jackvony EH, Wing RR. Effects of Internet behavioral counseling on weight loss in adults at risk for type 2 diabetes: a randomized trial. JAMA 2003; 289: 1833.

[37] Tate DF, Jackvony EH, Wing RR. A randomized trial comparing human e-mail counseling, computer-automated tailored counseling, and no counseling in an Internet weight loss program. Arch Intern Med 2006; 166: 1620.

[38] Tate DF, Wing RR, Winett RA. Using Internet technology to deliver a behavioral weight loss program. JAMA 2001; 285: 1172.

[39] Harvey-Berino J, Pintauro S, Buzzell P, Gold EC. Effect of internet support on the long-term maintenance of weight loss. Obes Res 2004; $12: 320$.

[40] Haugen HA, Tran ZV, Wyatt HR, Barry MJ, Hill JO. Using telehealth to increase participation in weight maintenance programs. Obesity (Silver Spring) 2007; 15: 3067.

[41] Shapiro JR, Bauer S, Hamer RM, Kordy H, Ward D, Bulik CM. Use of text messaging for monitoring sugar-sweetened beverages, physical activity, and screen time in children: a pilot study. J Nutr Educ Behav 2008; 40: 385. 
[42] Nardone G, Watzlawick P. The art of change. Strategic therapy and hypnotherapy without trance. San Francisco: Jossey-Bass 1993.

[43] Nardone G, Watzlawick P. Advanced brief therapy. Aronson. New Jersey 2004.

[44] Hay PP, Bacaltchuk J, Stefano S, Kashyap P. Psychological treatments for bulimia nervosa and binging. Cochrane Database Syst Rev 2009; 4: CD000562.

[45] Hay PJ, Bacaltchuk J. Bulimia nervosa. Clin Evid (Online) 2008; 2008: Pii, 1009.

[46] Hay PJ, Bacaltchuk J, Stefano S. Psychotherapy for bulimia nervosa and binging. Cochrane Database Syst Rev 2004; 3: CD000562.

[47] Hay PJ, Bacaltchuk J. Psychotherapy for bulimia nervosa and binging. Cochrane Database Syst Rev 2003; 3: CD000562.

[48] Hay PJ, Bacaltchuk J. Psychotherapy for bulimia nervosa and binging. Cochrane Database Syst Rev 2001; 3: CD000562.

[49] Hay PJ, Bacaltchuk J. Psychotherapy for bulimia nervosa and binging. Cochrane Database Syst Rev 2000; 4: CD000562.

[50] Cooper HM. Meta-analysis and the integrative research review. In: Hendrik C, Clark MS, Eds. Research methods in personality and social psychology. Newbury Park: Sage, 1990. p. 142.

[51] Ogden J, Hills L. Understanding sustained behavior change: the role of life crises and the process of reinvention. Health (London) 2008; 12(4): 419-37.

[52] Ogden J, Stavrinaki M, Stubbs J. Understanding the role of life events in weight loss and weight gain. Psychol Health Med 2009; 14: 239.

[53] Ryan RM, Patrick H, Deci EL, Williams GC. Facilitating health behaviour change and its maintenance: Interventions based on SelfDetermination Theory. Eur Health Psychol 2008; 10: 2.

[54] Perone R. SCID I/P DSM IV-TR Research Version 2002

[55] Chiappelli M, Lo Coco G, Gullo S, Bensi L, Prestano C. The outcome questionnaire 45.2. Italian validation of an instrument for the assessment of psychological treatments. Epidemiol Psichiatr Soc 2008; 17: 152 .

[56] Lo Coco G, Chiappelli M, Bensi L, Gullo S, Prestano C, Lambert MJ. The factorial structure of the Outcome Questionnaire-45: a study with an Italian sample. Clin Psychol Psychother 2008; 15: 418.

[57] Lambert MJ, Hansen NB, Umphress V, et al. Administration and scoring manual for the Outcome Questionnaire (OQ 45.2). Stevenson, MD: American Professional Credentialing Services LLC, 1996.

[58] Hatfield DR, Ogles BM. The use of outcome measures by psychologists inclinical practice. Professional Psychol: Res Pract 2004; 35: 485.

[59] Lackey NR, Wingate AL. The pilot study: one key to research success. Kans Nurse 1986; 61: 6.

[60] Wilson GT, Sysko R. Frequency of binge eating episodes in bulimia nervosa and binge eating disorder: Diagnostic considerations. Int J Eat Disord 2009; 42: 603.

[61] Riva G, Bacchetta M, Cesa G, et al. Is severe obesity a form of addiction? Rationale, clinical approach, and controlled clinical trial. Cyberpsychol Behav 2006; 9: 457.

[62] Castelnuovo G, Buselli C, De Ferrari R, et al. New tools in cybertherapy: the VEPSY web site. Stud Health Technol Inform 2004; 99: 15.

[63] Riva G, Botella C, Castelnuovo G, Gaggioli A, Mantovani F, Molinari E. Cybertherapy in practice: the VEPSY updated project. Stud Health Technol Inform 2004; 99: 3.

[64] Riva G, Alcaniz M, Anolli L, et al. The VEPSY Updated Project: clinical rationale and technical approach. Cyberpsychol Behav 2003; 6: 433 .

[65] Castelnuovo G, Gaggioli A, Mantovani F, Riva G. From psychotherapy to e-therapy: the integration of traditional techniques and new communication tools in clinical settings. Cyberpsychol Behav 2003; 6: 375.

[66] Gaggioli A, Mantovani F, Castelnuovo G, Wiederhold B, Riva G. Avatars in clinical psychology: a framework for the clinical use of virtual humans. Cyberpsychol Behav 2003; 6: 117.

[67] Castelnuovo G. No medicine without psychology: the key role of psychological contribution in clinical settings. Front Psychol Clin Settings. 2010; 1: 4; doi: 10.3389/fpsyg.2010.00004.

(C) Castelnuovo et al.; Licensee Bentham Open.

This is an open access article licensed under the terms of the Creative Commons Attribution Non-Commercial License (http://creativecommons.org/licenses/by-nc/3.0/) which permits unrestricted, non-commercial use, distribution and reproduction in any medium, provided the work is properly cited. 